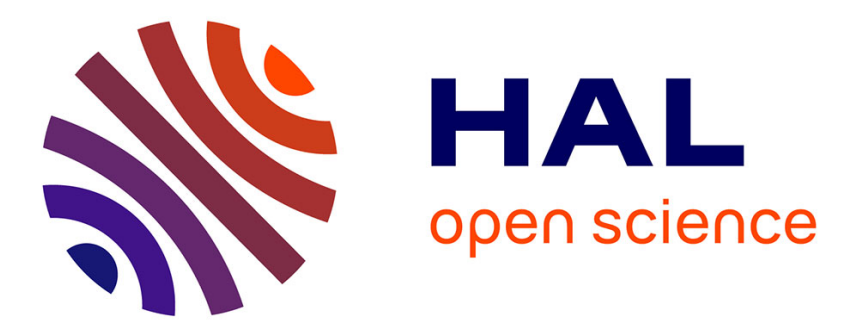

\title{
Bias on absolute luminosity measurements at the ILC from beam-beam space charge effects
}

\author{
Cécile Rimbault, P. Bambade, K. Mönig, D. Schulte
}

\section{To cite this version:}

Cécile Rimbault, P. Bambade, K. Mönig, D. Schulte. Bias on absolute luminosity measurements at the ILC from beam-beam space charge effects. 2007 Particle Accelerator Conference (PAC'07), Jun 2007, Albuquerque, United States. pp.3907-3909. in2p3-00200099

\section{HAL Id: in2p3-00200099 https://hal.in2p3.fr/in2p3-00200099}

Submitted on 20 Dec 2007

HAL is a multi-disciplinary open access archive for the deposit and dissemination of scientific research documents, whether they are published or not. The documents may come from teaching and research institutions in France or abroad, or from public or private research centers.
L'archive ouverte pluridisciplinaire HAL, est destinée au dépôt et à la diffusion de documents scientifiques de niveau recherche, publiés ou non, émanant des établissements d'enseignement et de recherche français ou étrangers, des laboratoires publics ou privés. 
LAL/RT 07-14

EUROTeV-Report-2007-055

November 2007

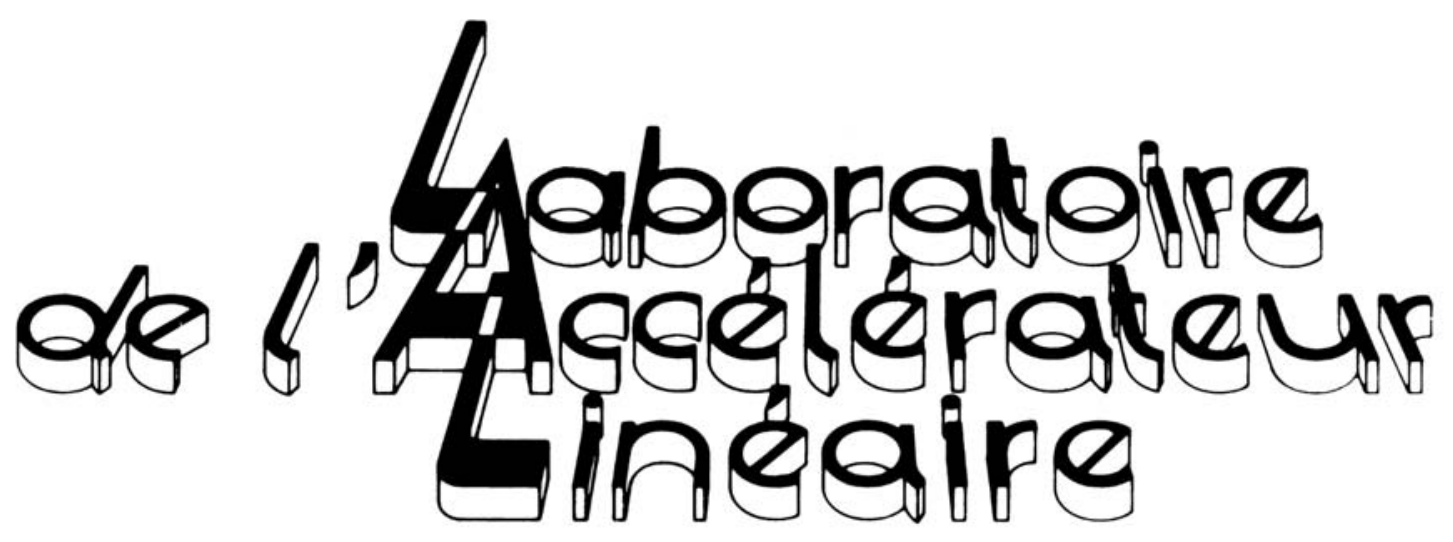

Bias on Absolute Luminosity Measurements at the ILC from Beam-Beam Space Charge Effects

\author{
C. Rimbault, P. Bambade \\ K. Mönig \\ DESY, Zeuthen, Germany \\ D. Schulte \\ CERN, Geneva, Switzerland
}

LAL, Univ Paris-Sud, CNRS/IN2P3, Orsay, France

Contribution to "PAC'07"

Albuquerque, New Mexico, USA, 25-29 June 2007

\section{U.M.R \\ de}

l'Université Paris-Sud

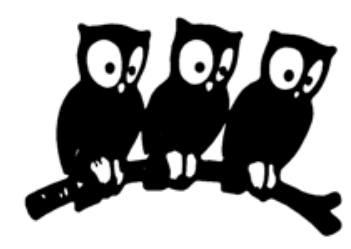

Institut National de Physique Nucléaire et de Physique des Particules du CNRS 
LAL/RT 07-14

EUROTeV-Report-2007-055

November 2007

\title{
Bias on Absolute Luminosity Measurements at the ILC from Beam-Beam Space Charge Effects
}

\author{
C. Rimbault, P. Bambade \\ LAL, Univ Paris-Sud, CNRS/IN2P3, Orsay, France \\ K. Mönig \\ DESY, Zeuthen, Germany \\ D. Schulte \\ CERN, Geneva, Switzerland
}

\begin{abstract}
This note summarizes the results of the first study done to quantify the impact of beam-beam effects on the precision luminosity measurement at the International Linear Collider, using GUINEA-PIG, a beam-beam interaction simulation tool.
\end{abstract}




\section{Introduction}

A way to determine luminosity at the International Linear Collider (ILC) is to measure the event rate of the Bhabha scattering process in a finely segmented calorimeter (LumiCal) at very low polar angles in the very forward region of the ILC detector. An absolute precision between $10^{-4}$ and $10^{-3}$ is needed for a number of key physics measurements $[1,2]$. Besides theoretical uncertainties on the cross section of the Bhabha process [3] and different experimental errors when identifying Bhabha events in the LumiCal [4], the very strong beam-beam space charge effects which characterise the ILC $e^{+} e^{-}$collisions can lead to large biases in the counting rate. These must be corrected accurately enough not to limit the precision of the luminosity measurement.

A first study of such biases has been performed using the following procedure [5]. A sample of Bhabha events is first produced with BHLUMI, a multiphoton Monte-Carlo event generator for small-angle Bhabha scattering [6] providing four-momenta of outgoing electron, positron and photons. The center-of-mass energy is $500 \mathrm{GeV}$ and the scattering angles are generated in the range $25 \mathrm{mrad}<\theta<90 \mathrm{mrad}$.

The four-momenta of the two charged final state particles of a generated event are then read into GUINEA-PIG [7], a beam-beam interaction simulation tool, and associated to one of the $e^{+} e^{-}$interactions occuring during the simulated bunch collision. GUINEAPIG computes the subsequent electromagnetic transport through the remaining part of the colliding bunch and the distribution of the resulting deflection is then obtained.

\section{Effect of Beam-Beam Space Charge on Bhabha Scattering}

Prior to the hard Bhabha scattering, the interacting particles are likely to have been deflected by the space charge of the opposite bunch and their energies reduced due to the emission of beamstrahlung. To take into account the cross section dependance with $s$, the probability used to produce Bhabha scattering events during the beam-beam collision is rescaled by $s / s^{\prime}$, where $s^{\prime}$ is the effective centre-of-mass energy. The fourvectors of the Bhabha event particles are also rescaled by $\sqrt{s^{\prime} / s}$, to satisfy energy and momentum conservation, as well as boosted from the centre-of-mass system of the two interacting particles to the laboratory frame. Finally the coordinate system is rotated to take into account the deflection angles of the interaction particles in the initial state. Just like the initial state radiation present in the initial sample generated with BHLUMI, the beamstrahlung emissions often occur asymmetrically, with either the electron or the positron loosing most of the energy. Hence the acollinearity of the final state can be significantly enhanced.

The final state particles scattered in the acceptance of the LumiCal following a Bhabha interaction can cross a significant part of the opposite bunch. They can thus be focused by the electromagnetic field from the corresponding space charge. In the GUINEAPIG simulation, the existing procedure to track secondary charged particles can be used conveniently to predict both the final deflection angle and any additional radiation. 
The resulting changes in scattering angles are displayed in Figure 1, where the differences between the initial polar angles before including the electromagnetic deflection, $\theta_{1}$ and the final ones including it, $\theta_{2}$ are shown as a function of $\theta_{1}$. The typical magnitudes of the induced electromagnetic deflections are a few $10^{-2} \mathrm{mrad}$, with the largest values at the lower edge of the LumiCal acceptance. Small energy losses due to radiation are also found.

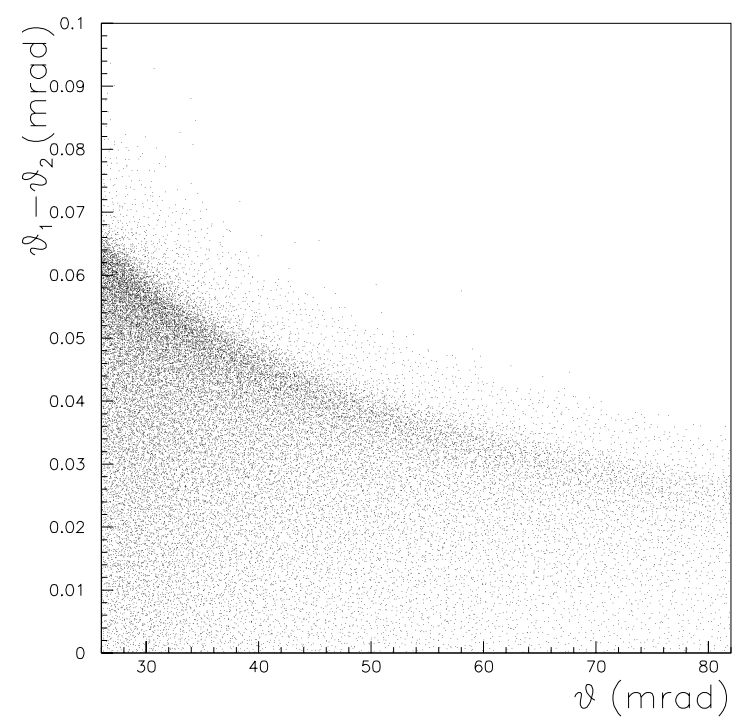

Figure 1: Change in Bhabha scattering final state polar angle due to the deflection induced by the space charge of the opposite bunch as a function of the polar angle at production.

Both the beamstrahlung radiation and electromagnetic deflection effects described above lead to a suppression of the Bhabha counting rate in the defined experimental acceptance in comparison to the theoretically predicted one. This BHabha Suppression Effect (BHSE) can be expressed as:

$$
\text { BHSE }=\frac{N_{\text {final }}-N_{\text {init }}}{N_{\text {init }}},
$$

where $N_{\text {init }}$ and $N_{\text {final }}$ are the numbers of Bhabha events selected within the specified cuts, respectively before and after including the different transformations to take into account effects from the beam space charge.

Because beamstrahlung radiation emitted prior to the hard Bhabha scattering enhances the acollinearity between the two final state charged particles, assymetrical angular cuts [4] can help to minimise the BHSE and the resulting biases, for instance:

$$
\begin{aligned}
30 \mathrm{mrad} & <\theta_{+/-}<75 \mathrm{mrad} \\
26.2 \mathrm{mrad} & <\theta_{-/+}<82 \mathrm{mrad} .
\end{aligned}
$$

applied randomly, respectively to either the electron and positron, or vice-versa. 
Similarly assymetrical cuts on the energies are also advantageous. Choosing a global energy cut based on the sum of the final state energies:

$$
E_{-}+E_{+}>0.8 \sqrt{s}
$$

as well as the angular cut in 2, the magnitude of BHSE is of the order of -0.015 for the ILC Nominal beam parameter set, two thirds of which is due to beamstrahlung emissions. Without such assymetrical cuts, the BHSE bias in enhanced by a factor of about three.

\section{Sensitivity to Beam Parameters}

Both the beamstrahlung emissions and the electromagnetic deflection vary with the bunch parameters and the energy of the collision, and thus also the corresponding biases on the integrated luminosity. The reconstruction of the luminosity spectrum, based on the scattered Bhabha angles [8], provides a good way to measure the amount of beamstrahlung and thus to predict the corresponding contribution to the bias, because the electromagnetic deflections do not modify signicantly this spectrum [5]. However, this also means that such a method does not allow probing effects from electromagnetic deflections experimentally.

In the following, the sensitivity of the BHSE on bunch parameters at the collision point and the residual dependancies which can be expected after correcting for the main contribution arising from beamstrahlung are discussed.

During operation of the ILC, bunch sizes and alignment at the interaction point can be expected to vary over time, due to dynamical imperfections in the acceleration and optical transport and through injection errors. Dedicated feedback control loops are included in the ILC design to maintain the bunch parameters constant within appropriate tolerances [9]. In this context, it is interesting to evaluate the precision needed on the knowledge of the main parameters to keep the BHSE within a given level of accuracy. It was found that the BHSE only depends on both the horizontal size $\sigma_{x}$ (see Fig. 2) and the bunch length $\sigma_{z}$. This can be understand because these parameters are directly related to the beamstrahlung emission. From the non-linear curves can be estimated the uncertainty on the BHSE resulting from a given precision assumed on the knowledge of $\sigma_{x}$ and $\sigma_{z}$. Sine the main uncertainty on the BHSE arises through the contribution from beamstrahlung, this part can in principle be measured and corrected from the luminosity spectrum reconstruction, the residual uncertainty which remains comes from the contributions of electromagnetic deflections. From the curves it can be deduced that to limit the error on the BHSE from this part to about $10^{-3}$, a precision at the $20 \%$ level is needed on the knowledge of $\sigma_{z}$ and $\sigma_{x}$.

ILC should allow physics runs initially for energies between the $\mathrm{Z}$ boson mass and $\sqrt{s}=500 \mathrm{GeV}$ [10]. In this energy range beam-beam effect are strongly modified.

The dependence of BHSE with energy is shown in Figure 3, in the assumption that all optical parameters and the bunch length and intensity are kept constant. At the lower end of the energy range, the beamstrahlung contribution to the BHSE decreases to a 
few $10^{-4}$. But on the other hand, electromagnetic deflections become rapidly stronger, dominating the bias below $400 \mathrm{GeV}$.

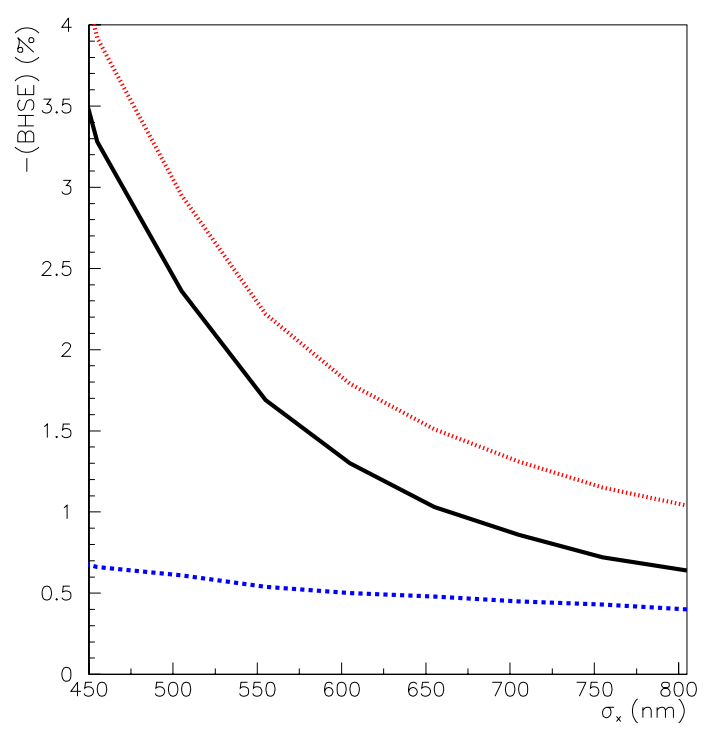

Figure 2: Bhabha suppression effect as a function $\sigma_{x}$. The contributions due to beamstrahlung (full line) and electromagnetic deflections (dashed line) are shown as well as the combined effect (top dotted line).

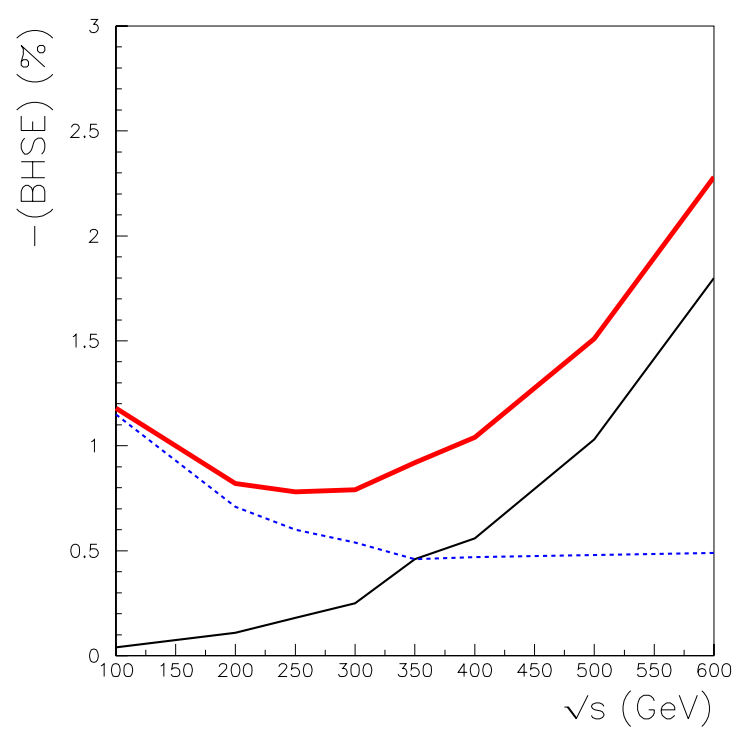

Figure 3: Bhabha suppression effect as a function of energy. The contributions due to beamstrahlung (full line) and electromagnetic deflections (dashed line) are shown as well as the combined effect (top dotted line). 


\section{Conclusion}

In this study, it was shown, for the first time, that taking into account beam-beam interactions, the precise knowledge of the theoretical cross section is no longer sufficient to measure the luminosity with high precision (better than $10^{-3}$ ), because strong collective effects modify drastically the kinematics of the Bhabha process. The first changes come from beamstrahlung, which induces a perturbation of the initial state. A second set of transformations arises from electromagnetic deflections which modify the kinematical phase space of the scattered Bhabha events. Using the beam-beam interaction simulation tool GUINEA-PIG, it was estimated that the bias induced on the luminosity measurement at low angle is about -0.015 for the Nominal beam parameter set. Two thirds of this bias are due to beamstrahlung emissions.

Both beamstrahlung emissions and electromagnetic deflections vary with the bunch length, $\sigma_{z}$, the horizontal size, $\sigma_{x}$, and the energy of the collision, and hence also the resulting biases on the integrated luminosity. Reconstructing the luminosity spectrum from the scattered Bhabha angles provides a good way to measure the amount of beamstrahlung, and thus to predict the corresponding contribution to the bias, because the electromagnetic deflections do not modify significantly this spectrum. Once this is done, controlling $\sigma_{x}$ and $\sigma_{z}$ at the $20 \%$ level around the nominal values is enough to limit the remaining contribution to the luminosity bias, from the deflections, to $10^{-3}$.

Unlike the beamstrahlung emissions, no direct way to control experimentally the bias from the electromagnetic deflections is available from the data. Nevertheless, measurements of the beam angular divergence in the beam extraction line could be a way to monitor it. Further studies are needed on this point. Numerical simulations such as GUINEA-PIG [7] and CAIN [11] are essential estimation tools in all of this. A practical implementation to compute these effects is available at [12] and can be used for further studies.

In particular, physics running on the $\mathrm{Z}$ boson resonance is planned as an option in the ILC program (the GigaZ option). The accuracy on the luminosity is specified to be $10^{-4}$ in this case, while the bias from the electromagnetic deflections is at least a hundred times larger. Further more complete studies will then be particularly important, to explore all relevant dependencies and to devise data-driven correction methods.

\section{Acknowledgement}

This work is supported by the Commission of the European Communities under the $6^{\text {th }}$ Framework Programme "Structuring the European Research Area", contract number RIDS-011899.

\section{References}

[1] TESLA Technical Design Report, DESY 2001-011, March 2001. 
[2] The Forward Calorimetry Group, "R\&D for the ILC-Detector: Instrumentation of the Very Forward Region", DESY PRC R\&D 02/01, April 2006.

[3] S. Jadach, "The Physics Case for the International Linear Collider", june 2003. [hep-ph/0306083]

[4] A. Stahl, "Luminosity Measurement via Bhabha Scattering: Precision Requirements for the Luminosity Calorimeter" LC-DET-2005-004, 2005.

[5] C. Rimbault, P. Bambade, K. Mönig, D. Schulte, "Impact of beam-beam effects on precision luminosity measurements at the ILC", EUROTeV-Report-2007-017, to be published.

[6] S. Jadach, W. Placzek, E. Richter-Was, B.F.L. Ward, Z. Was, "Upgrade of the Monte-Carlo program BHLUMI for Bhabha scattering at low angles to version 4.04", june 1996, CERN-TH/96-158. UTHEP-96-0601.

[7] D. Schulte, Ph. D. Thesis, University of Hamburg 1996. TESLA-97-08.

[8] K. Mönig, "Measurement of the Differential Luminosity using Bhabha events in the Forward-Tracking region at TESLA", LC-PHSM-2000-60-TESLA, december 2000.

[9] International Linear Collider Reference Design Report, first draft, february 2007. http://media.linearcollider.org/rdr_draft_v1.pdf

[10] A. Djouadi, J. Lykken, K. Mönig, Y. Okada, M. Oreglia, S. Yamashita, "The Physics Case for the International Linear Collider", Frozen draft for the ACFA meeting in Beijing, February 2007.

http://www-zeuthen.desy.de/ILC/dcr_physics/dcr_beijing.pdf

[11] K. Yokoya, User's Manual of CAIN - Version 2.3, october 2001. http://www-acc-theory.kek.jp/members/cain/default.html

[12] http://flc.web.lal.in2p3.fr/mdi/BBSIM/bbsim.html 